\title{
Sleep and Shift Work - Less Than Ideal Partners
}

\author{
Mário J. Santos*; Diogo Almeida*; Júlio Santos*; Teresa Maia* \\ * Psychiatry Department - Hospital Prof. Doutor Fernando Fonseca
}

\section{Background}

The labor demand of modern life forced the emergence of shift work in various professional areas, like law enforcement, fire safety and healthcare. Shift work has been defined as occurring when at least a portion of the shift occurs between 7 PM and 6 AM. In some countries, almost $20 \%$ of workers work some form of non-traditional shift. Several studies have looked at the health consequences of this type of work, ranging from cognitive complaints to cancer, as well as diminished quality of life.

\section{Objectives}

Review Shift Work Disorder (SWD) and the effects that shift work has on the quality and sleep pattern of individuals.

\section{Materials and Methods}

Brief review of literature available at the Pubmed/Medline database, searched using the keywords "shift work", "sleep" and "shift work disorder".

\section{Shift Work Disorder (SWD)}

SWD is a circadian rhythm sleep disorder,, that happens as a result of shift work, characterized by excessive sleepiness during the desired waking period and/or insomnia when sleep is allowed.

Prevalence of SWD is understudied, but some evidence suggests that more than one in five shift workers suffers from it. These workers experience a desynchronization of the processes that regulate sleep and wakefulness.

Shift workers demonstrate significant variability in tolerance for and adaptation to shift work. Workers who start a new shift will often suffer from fatigue and later insomnia. While most of them will be able to make the transition after a few days, some workers may not be able to. The mechanisms behind this being poorly understood.

\section{Clinical Assessment}

Clinical interview, including a detailed sleep history, a careful review of the general and psychiatric medical history and medication profile are of paramount importance. Sleep logs and actigraphy may also be helpful. It is essential to rule out other known causes of excessive sleepiness, including sleep disorders such as obstructive sleep apnea and narcolepsy.

\section{Management}

A central issue when talking SWD is to reduce the circadian misalignment in order to enhance adaptation to the shift work schedule. One of the most investigated strategies in this sense is bright light exposure, but as proven difficult to translate to a real world setting. Other strategy could be the administration of melatonin before sleep, although some studies state it to be less helpful than to other sleep disorders.

For improving sleep, behavioral approaches are usually recommended, as typical sedative hypnotics and insomnia pharmacotherapies raise performance and safety concerns among patients with SWD.

One of the main strategies used by individuals to ensure an adequate work performance is the consumption of caffeine. Other agents like amphetamines and methylphenidate were studied, but there are many concerns over abuse. Modafinil and armodafinil are FDA-approved medications for improving nocturnal wakefulness in SWD patients.

\section{Organizational Approach}

Shifts have multiple distributions and periodicities. There are permanent or rotating shifts. The latter can be rapid shifting (multiple schedule changes per week) or slow shifting (e.g. multiple weeks with the same shift). When night shifts are unavoidable, permanent nights or clockwise or forward-rotating nights seem preferable. Rapid shifting have been associated with improved outcomes such as better sleep quality and reduced fatigue. Due to substantial inter-individual variability, a "one-rule fits all" approach is unlikely to maximize outcomes.

\section{Conclusions}

The proliferation of shift work leads to more frequent occurrence of sleep disorders. These disorders cause insomnia and excessive daytime sleepiness, with consequent impact on health and quality of life. As shift work will remain a vital component of the modern economy, professionals should be aware of its implications in clinical practice.

References

- Abbott SM, Reid KJ, Zee PC. Circadian Rhythm Sleep-Wake Disorders. Psychiatr Clin N Am (2015). 38(4):805-823.

- Costa G, Anelli MM, Castellini G, Fustinoni S, Neri L. Stress and sleep in nurses employed in " $3 \times 8$ " and "2 X 12" fast rotating shift schedules. Chronobiology International (2014). 31(10):1169-1178.

- Flo E, Pallesen S, Åkerstedt T, el al. Shift-related sleep problems vary according to work schedule. Occup Environ Med (2013). 70:238-245.

- Wickwire EM, Geiger-Brown J, Scharf SM, Drake CL. Shift Work and Shift Work Sleep Disorder: Clinical and Organizational Perspectives. CHEST (2017). 151(5):1156-1172 